\title{
Neural specificity for grammatical operations is revealed by content-independent fMR adaptation
}

\author{
Kevin A. Shapiro ${ }^{1,2,3}$, Lauren R. Moo ${ }^{3}$ and Alfonso Caramazza ${ }^{1,4}$ * \\ 1 Department of Psychology, Harvard University, Cambridge, MA, USA \\ 2 Department of Medicine, Children's Hospital Boston, Boston, MA, USA \\ ${ }^{3}$ Department of Neurology, Massachusetts General Hospital, Boston, MA, USA \\ ${ }^{4}$ Center for Mind/Brain Sciences, University of Trento, Rovereto, Italy
}

Edited by:

Kristof Strijkers, University of

Barcelona, Spain

Reviewed by:

Ian G. Roberts, University of

Cambridge, UK

Laura Menenti, Max Planck Institute

for Psycholinguistics, Netherlands

*Correspondence:

Alfonso Caramazza, Department of

Psychology, Harvard University, 33

Kirkland Street, Cambridge, MA

02138, USA.

e-mail: caram@wjh.harvard.edu
The ability to generate novel sentences depends on cognitive operations that specify the syntactic function of nouns, verbs, and other words retrieved from the mental lexicon. Although neuropsychological studies suggest that such operations rely on neural circuits distinct from those encoding word form and meaning, it has not been possible to characterize this distinction definitively with neuroimaging. We used functional magnetic resonance imaging (fMRI) to show that a brain area engaged in a given grammatical operation can be identified uniquely by a monotonic decrease in activation as that operation is repeated. We applied this methodology to identify areas involved selectively in the operation of inflection of nouns or verbs. By contrast, areas involved in processing word meaning do not show this monotonic adaptation across stimuli. These results are the first to demonstrate adaptation in the fMR signal evoked not by specific stimuli, but by well-defined cognitive linguistic operations.

Keywords: nouns, verbs, grammatical class, inflection, fMRI, adaptation

\section{INTRODUCTION}

Several reports in the neuropsychological literature have described patients whose patterns of brain damage have resulted in disproportionate difficulty using either nouns or verbs in speech (Miceli et al., 1984; McCarthy and Warrington, 1985; Caramazza and Hillis, 1991; Damasio and Tranel, 1993; Daniele et al., 1994). In many of these cases, the apparent grammatical deficit may reflect the destruction of neurons involved in representing features of entities or actions, the prototypical referents of nouns and verbs, respectively. Thus, patients with lesions affecting higherorder visual association areas in the temporal lobe tend to have more difficulty with object words (produced as nouns), while those with lesions in premotor areas fare more poorly in naming action words (verbs) (Damasio and Tranel, 1993; Daniele et al., 1994; Bak et al., 2001; Luzzatti et al., 2006).

Interestingly, there are patients whose impairment seems specifically to affect the use of words in a particular grammatical context, while access to word meaning is relatively unaffected (Shapiro et al., 2000; Berndt et al., 2002; Shapiro and Caramazza, 2003; Laiacona and Caramazza, 2004; Crepaldi et al., 2006). Some patients are unable to produce even nonsense words marked inflectionally as nouns (e.g., the toves), although they are able to produce the same strings as verbs (he toves) (Shapiro et al., 2000); other patients exhibit the mirror dissociation (Shapiro and Caramazza, 2003; Laiacona and Caramazza, 2004). These cases support the idea that there are neuroanatomically dissociable regions involved in the processing of nouns and verbs as grammatical objects.

Limited anatomical comparisons suggest that neural regions in the left prefrontal cortex may be crucial to support the distinction between nouns and verbs in grammatical processing (Shapiro et al., 2001; Shapiro and Caramazza, 2003; Cappelletti et al., 2008). However, category-selective deficits are present in only a subset of patients with left prefrontal lesions. Most patients with lesions affecting the left frontal operculum (Broca's area) and underlying structures, as well as the striate nuclei of the basal ganglia (Longworth et al., 2005), do have some degree of impairment in producing morphological affixes (Goodglass, 1973; Dronkers et al., 2000). These impairments often do not respect grammatical category distinctions (Miceli et al., 1989), but may selectively affect certain kinds of morphological operations for words of all categories, like the production of regular inflectional affixes (Marslen-Wilson and Tyler, 1997; Ullman et al., 1997; Faroqi-Shah and Thompson, 2004). Such patterns suggest that distinct cortical regions are engaged in processing morphological information within and across grammatical categories.

Studies using repetitive transcranial magnetic stimulation (rTMS) in unimpaired subjects have supported this notion, and have helped to identify anatomically dissociable circuits within the left prefrontal cortex that may be involved in category-specific and category-general morphological operations. Two studies have demonstrated that grammatical operations involving verbs, but not nouns, are disrupted selectively by stimulation to a circumscribed portion of the left mid-frontal cortex that is superior to Broca's area and well anterior to motor planning areas (Shapiro et al., 2001; Cappelletti et al., 2008). By contrast, stimulation to an adjacent portion of Broca's area affects noun and verb production equally (Cappelletti et al., 2008). One interpretation of this pattern is that the left mid-frontal region is important for handling abstract grammatical (morphosyntactic) information that pertains to verbs (Shapiro et al., 2001; Shapiro and Caramazza, 
2003; Cappelletti et al., 2008), while Broca's area is important for category-general morphological processes, and perhaps specifically for the phonological realization of morphological features (Kean, 1978; Obler et al., 1999).

Functional neuroimaging methods have also been applied in an attempt to elucidate the neural circuits involved in the grammatical processing of nouns and verbs. Interestingly, these experiments for the most part have not provided evidence to corroborate the anatomical distinction that is strongly implied by neuropsychological and TMS studies. Imaging studies have shown some differences in activation evoked by nouns and verbs in the left inferior prefrontal cortex, but these differences tend to correlate with the linguistic complexity of inflected noun and verb stimuli, rather than with categorical grammatical operations as such (Perani et al., 1999; Tyler et al., 2004; Sahin et al., 2006; Longe et al., 2007). When noun and verb stimuli are well matched, as in the case of homophones, no differences between categories are observed in Broca's area (Tyler et al., 2008; Burton et al., 2009).

Several studies have shown anatomical dissociations between nouns and verbs in other regions, particularly in the left middle temporal lobe (Perani et al., 1999; Shapiro et al., 2006; Yokoyama et al., 2006; Palti et al., 2007; Bedny et al., 2008; Liljeström et al., 2008; Burton et al., 2009; Khader et al., 2010). These studies have employed a wide variety of tasks involving both production and comprehension, including phrase completion (Shapiro et al., 2006; Khader et al., 2010), picture naming (Liljeström et al., 2008), lexical decision (Perani et al., 1999; Yokoyama et al., 2006), semantic judgment (Palti et al., 2007; Bedny et al., 2008), and grammatical judgment (Palti et al., 2007; Burton et al., 2009). Regardless of task, the observed dissociations in areas outside the left prefrontal cortex are most plausibly related to differences in word meaning - even when subjects perform tasks that do not involve semantic processing, or specifically emphasize the manipulation of grammatical information.

The mixed results of functional imaging studies on the question of noun-verb differences may be attributable in part to the inherent difficulty of constructing an imaging experiment to isolate differences in a single processing component among many that are engaged during even a simple inflection task. One recent review has gone so far as to suggest that the neural circuits underlying noun and verb processing, if they exist, are irresoluble using current imaging methods (Crepaldi et al., 2011). A potential means of circumventing this problem may lie in the properties of the dependent variable in imaging studies in the case of fMRI studies, the blood oxygen level dependent (BOLD) signal. Standard event-related fMRI contrast analyses assume that the amplitude of the BOLD signal evoked by a given task remains constant over time. Recently, however, numerous investigators have shown that when a particular stimulus is repeated, the activation associated with processing that stimulus is reduced (Henson et al., 2000; Grill-Spector et al., 2006). This effect, known as fMR adaptation or repetition suppression (GrillSpector et al., 2006), has been exploited productively as a means of making inferences about the specificity of brain regions for processing different kinds of representational content in various domains, including object naming (van Turrennout et al., 2003), semantic and phonological processing (Wagner et al., 2000; Gold et al., 2005), and lexical and syntactic processing (Menenti et al., 2011).

Here we are interested in identifying not brain areas that process a particular kind of content, but areas specialized for performing particular sets of operations, namely, the context-dependent morphosyntactic marking of nouns and verbs. Generalizing from earlier studies demonstrating stimulus-specific fMR adaptation, we reasoned that neural circuits engaged in particular cognitive operations of this sort may show operation-specific continuous adaptation during repetition of a task regardless of the content of individual stimuli. There is some evidence that brain regions may show a decrement in activity when a task is repeated using novel stimuli (Martin et al., 1997; Toni et al., 1998). Toni et al. (1998), for example, observed that there was a decrease in the magnitude of the BOLD signal over time in parts of the prefrontal, premotor, and parietal cortex as subjects learned simple motor sequences. Our hypothesis can be viewed as an extension of this finding.

We therefore designed an fMRI experiment in which subjects were required to complete simple phrases with grammatically appropriate forms of nouns and verbs and looked for brain regions demonstrating a linear decrement in activity for morphologic operations involving nouns or verbs. In the first session of the experiment, the stimuli consisted of meaningless pseudowords whose grammatical category was indicated solely by phrasal context (he zeeks, they. ..; many glushes, one...). In the second session, the stimuli were real nouns and verbs (one loss, many...; he sings, they...). The use of pseudowords underscores the emphasis on identifying target words according to their syntactic roles, rather than on retrieval of lexical-semantic information.

Half of the trials in each session required subjects to change the morphological form of the stimulus word; in the other half, subjects were required to produce the stimulus word in the same form. Thus, words were produced in both their inflected (zeeks) and unmarked forms (zeek), and the target form was not predictable from the stimulus phrase. Moreover, for half of the real and pseudowords in each category, the inflected form differed in syllabic structure from the unmarked form (e.g., glush/glushes). These manipulations were intended to ensure that contrasts in activation evoked by nouns and verbs could be interpreted as reflecting primarily grammatical operations, rather than processes at the level of word meaning or phonological form.

We constructed two factorial models for random effects analysis of the functional data. In the first model (the "activation model"), we used statistical parametric maps corresponding to conventional event-related contrasts, based on standard assumption that the amplitude of the hemodynamic response to an event (a noun or verb trial) does not vary over time. In the second model (the "adaptation model"), we used maps which were estimated assuming a linear decrease in the amplitude of sequential trials of a given event type (i.e., grammatical operation), no matter the form of the stimulus. Again, this differs from prior fMRI adaptation experiments, which have generally examined stable differences in activation between repeated and novel stimuli. Our choice of a linear function, as opposed to a higher-order function, was arbitrary. It was our intention simply to determine whether modeling a monotonic decrease in signal could capture important information about the neural correlates of grammatical processing. 
In this context, three strong predictions follow from the hypothesis that neural adaptation occurs for the cognitive operations involved in noun and verb inflection. The first is that adaptation for both noun and verb trials should be observed in brain regions known to be important for processing regular morphological inflection irrespective of grammatical category. These regions may include the left inferior prefrontal cortex and the striate nuclei of the basal ganglia (Ullman et al., 1997; Longworth et al., 2005), areas that are thought to constitute a circuit subserving regular inflectional affixation.

Although activation has been observed in these areas in neuroimaging experiments that have examined the processing of inflected words, such experiments have also shown activation in areas not specifically implicated in inflection in the aphasiological literature, including premotor areas that may be engaged in articulation (Sahin et al., 2006) and orbital prefrontal and temporal areas that may support semantic processes (Tyler et al., 2005; Sahin et al., 2006). These prior studies, like many other neuroimaging studies, have not been able to distinguish areas that are activated in a given contrast from areas that are specifically dedicated to or crucial for the task of interest. By contrast, we predict that grammatical operation dependent adaptation should not be expected in regions that handle information that varies between trials, including information about the phonological or semantic properties of individual stimulus items.

A final and crucial prediction is that grammatical categoryspecific adaptation for verb trials should be observed in the portion of the left middle frontal gyrus whose disruption with rTMS selectively interferes with the grammatical processing of verbs. (An analogous region engaged in grammatical processing of nouns has not yet been identified.) Such a finding would suggest that content-independent task or operation based adaptation effects can be used not only as a means of discriminating between areas activated by a task and areas dedicated to a task, but also as a sensitive means of identifying areas critical for the performance of well-defined cognitive operations.

\section{MATERIALS AND METHODS SUBJECTS}

Twenty-two right-handed native English speakers (11 male) participated in the experiment, which was approved by institutional review boards at Harvard University and Massachusetts General Hospital. Informed consent was obtained from all subjects, and the experiment was conducted according to the principles expressed in the Declaration of Helsinki.

\section{MATERIALS}

The stimuli consisted of 104 pseudowords in session 1 and 104 monosyllabic real words (52 nouns and 52 verbs) in session 2 . The real words were matched across categories for frequency (Francis and Kucera, 1982), length in phonemes, and length in letters. Nouns and verbs were also matched for the number of consonants in the codas of the words; moreover, half of the words in each category ended in sibilant consonants, while half did not. The latter two criteria were intended to ensure that noun and verb stimuli were matched with respect to the phonological complexity of morphologically inflected forms. Pseudowords were generated by modifying the initial phonemes of the real words (e.g., seek/zeek).

The behavioral paradigm was identical to that used in an earlier experiment (Shapiro et al., 2006). In brief, subjects were presented with a written stimulus phrase (e.g., he zeeks) for $1 \mathrm{~s}$, followed for $1 \mathrm{~s}$ by a cue phrase (they) indicating the form in which the subject should produce the word or pseudoword that appeared in the stimulus phrase. In the preceding example, the subject would have been required to say "zeek." Trials were separated by the appearance of a fixation cross for intervals of varying duration between 2 and $6 \mathrm{~s}$. We have previously demonstrated that this task does not produce differences in reaction time by category (noun vs. verb) or lexical status (real word vs. pseudoword) for stimuli matched in the manner described above (Shapiro et al., 2006). In the current experiment, each stimulus word appeared in two trials (only one of which required the word to be produced in the inflected form), so that there were 208 unique trials per session.

Prior to the fMRI session, subjects completed a brief training session on a laptop computer. During the training session the subjects were instructed to respond aloud, but without moving their jaws, as if they were practicing ventriloquy. This method has minimizes head motion artifacts that result from the production of spoken responses inside the scanner. The training session used a set of pseudowords that did not occur in the experiment. In the scanner, the stimuli were projected using a collimating lens onto a mirror affixed to the head coil at a comfortable viewing distance. Responses were monitored by intercom, but were not recorded.

\section{DATA ACQUISITION AND PRE-PROCESSING}

Whole brain functional images were acquired on a $3-\mathrm{T}$ Siemens MR scanner using a T2*-weighted echoplanar imaging sequence (repetition time $(\mathrm{TR})=2 \mathrm{~s}$, echo time $(\mathrm{TE})=30 \mathrm{~ms}$, flip angle $=90^{\circ}, 64 \times 64$ acquisition matrix, 33 slices, no gap, $4 \mathrm{~mm}^{3}$ isotropic resolution). Pre-processing of the functional images in SPM5 included slice-time correction, realignment and unwarping, normalization to a standard template for echoplanar imaging sequences, resampling at $2 \mathrm{~mm}^{3}$, spatial smoothing with an $8-\mathrm{mm}^{3}$ full width at half maximum Gaussian kernel, and application of a temporal high-pass filter.

For all subjects, we also acquired high-resolution T1-weighted anatomical images $(\mathrm{TR}=30 \mathrm{~ms}, \mathrm{TE}=3.3 \mathrm{~ms}, 128$ slices, no gap, $1 \mathrm{~mm} \times 1 \mathrm{~mm} \times 1.33 \mathrm{~mm}$ resolution). The anatomical images were spatially coregistered using SPM5 and the automated segmentation algorithm included in this software package was used to generate maps of gray matter voxels, including cortical, subcortical, and cerebellar gray matter while excluding white matter and cerebrospinal fluid spaces. These maps were then averaged to generate a mean gray matter map, which was used for masking in analysis of the functional data (see below) and for purposes of display (figures show areas of functional activation projected on a surface rendering derived from the mean gray matter map).

\section{STATISTICAL MODELING}

Using the pre-processed functional images, we constructed firstlevel statistical models for each participant in which square waves representing onsets and durations of trials of each event type 
were convolved with a canonical hemodynamic response function, generating a basis function for each event type. There were four event types (real nouns, real verbs, pseudonouns, and pseudoverbs), in addition to fixation. The model also included two covariates to account for length in letters of the visual stimuli and the phonological complexity of the form being manipulated (sibilant or non-sibilant ending).

Crucially, for each event type we included an "adaptation" parameter modeling a linear decrement in the amplitude of the square wave function over time. This parameter simply made use of the option provided in SPM5 for first-order time modulation of an event type in a first-level model, predicting greater positive deviation from the average hemodynamic signal on earlier trials, and smaller positive deviation or a greater negative deviation (representing a relative suppression of activation) on later trials. In other words, it models a decrement in activation for a given trial type over time. The decrement is determined over a sequence of trials of the same type, irrespective of the position of a given trial within a series or block (see Figure $\mathbf{1}$ for an illustration). This is similar to the type of analysis described by Toni et al. (1998), except that in that paper, the authors modeled changes over time with polynomial functions up to the third order. We also did not remove low-frequency changes over time that are common to conditions, as we directly compared experimental conditions to each other - in which case common effects would be expected to cancel out - rather than to a baseline.

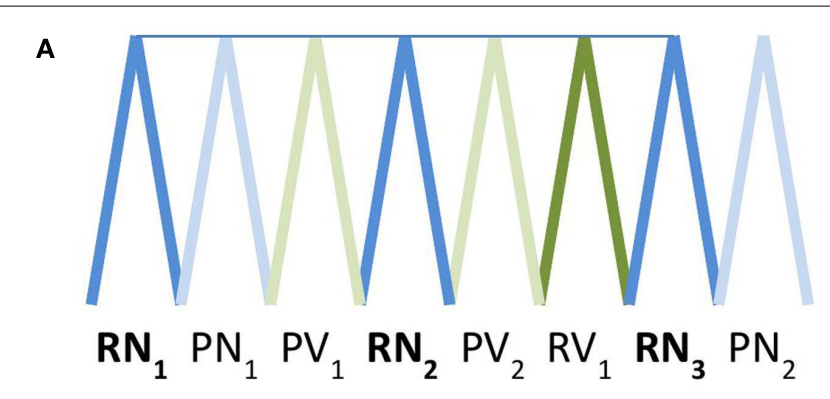

B

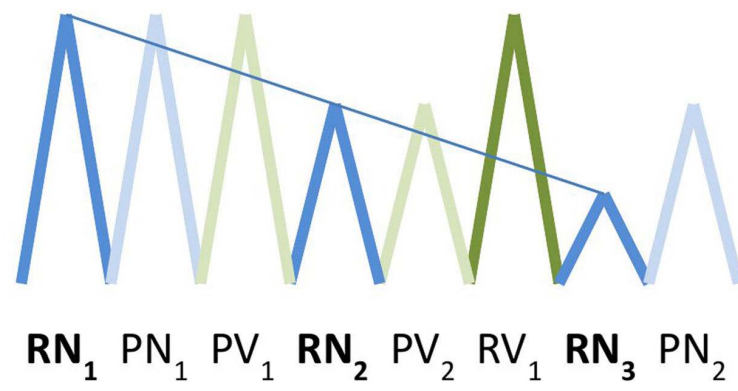

FIGURE 1 | Schematic depiction of basis functions used for modeling of activation (A) and adaptation (B). Square wave functions convolved with the standardized hemodynamic response function are represented here as triangle waves for ease of illustration. In the activation model, the amplitude of activation within a voxel for a given trial type was expected to remain constant over time. In the activation model, the amplitude of activation for a given trial type was expected to decrease in a linear fashion over successive trials. RN, real noun; RV, real verb; PN, pseudonoun; PV, pseudoverb.
We acknowledge that choosing a first-order function is a simplistic assumption both computationally and physiologically; we have no compelling reason to suppose that operation-specific adaptation is best modeled by a linear function as opposed to some other function, such as exponential decay. Indeed it is possible that the shape of the adaptation function differs in different brain regions, as in the study by Toni et al. (1998). In this sense the choice of a linear function can be regarded as a strong test of an underconstrained hypothesis. We return to this point in the Discussion.

After the first-level models including the basis functions and adaptation parameters for each event type were completed, we selected statistical parametric maps corresponding to $\beta$-weights of correlation between the predicted and observed hemodynamic response for nouns, verbs, pseudonouns, and pseudoverbs. We entered these into second-level ANCOVA models. Separate models were constructed using maps estimated using the standard eventrelated function and maps estimated using the linear adaptation parameter. In estimating the models, we applied a mask representing the mean distribution of gray matter voxels across subjects, as described in the Methods (Data Acquisition and Pre-Processing).

To obtain a general view of brain areas engaged by the task, we examined average effects using a voxel-wise type I error threshold of $\alpha=0.0005$, corrected for family-wise error. We applied an arbitrary cluster extent threshold of $k=80$ resampled voxels, equivalent in spatial extent to 10 original (non-resampled) voxels. In the activation model, the resulting statistical parametric map shows areas in which activity was correlated with the behavioral task, regardless of trial type, or position within the series. In the adaptation model, the corresponding map shows areas in which activity is correlated with the task and decreases for sequential trials of the same type, over all trial types.

For main effects of grammatical category in each model, we adopted a voxel-wise type I error threshold of $\alpha=0.005$ and used the cluster extent method to correct for multiple comparisons (Slotnick et al., 2003). Areas surpassing a corrected cluster-wise type I error threshold of $\alpha=0.05$ ( $k>56$ voxels) were selected for further analysis to determine the directionality of category-specific main effects and to test for interactions. For this post hoc analysis, we extracted subject wise first eigenvariate values for each significant cluster as an estimate of a region's hemodynamic response to processing nouns and verbs. These values were then entered into ANOVA models with lexical status and grammatical category as within-subjects repeated measures. A Bonferroni correction $(\alpha=0.05)$ was applied to account for comparisons across multiple areas. The values of the first eigenvariates corresponding to activation and adaptation for trials of each category within each significant cluster are plotted in Figure 2.

\section{RESULTS}

Using both the activation and adaptation fMRI analysis models, we aimed to identify areas with task-related effects irrespective of grammatical category, as well as areas showing selective effects for one grammatical operation.

\section{TASK GENERAL EFFECTS}

Using the activation model, we found that grammatical categorygeneral activation was most robust in a cortical network 


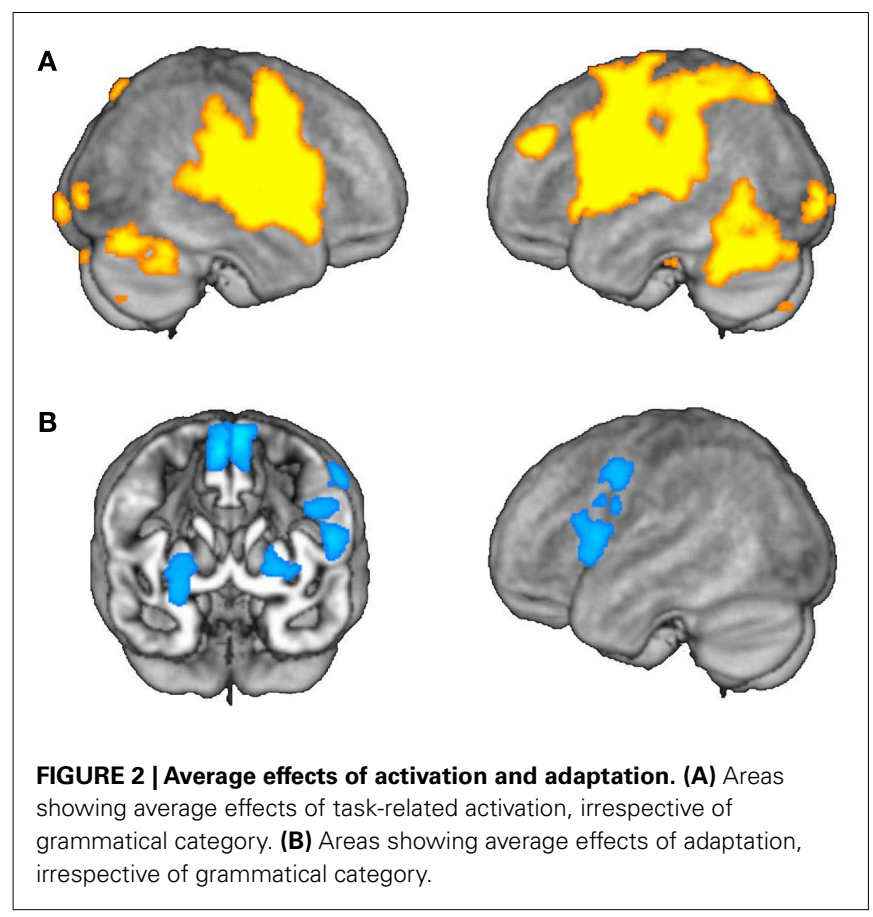

encompassing bilateral perisylvian and precentral regions and the anterior parts of the cingulate gyri, as well as the cerebellar hemispheres and bilateral subcortical nuclei (Table 1; Figure 2A). On the other hand, the adaptation model identified linear decreases in operation-related activation for both nouns and verbs primarily in the left inferior and posterior frontal cortex (including Broca's area) and in the lentiform nuclei and supplementary motor areas (SMAs) bilaterally (Table 2; Figure 2B).

\section{GRAMMATICAL CATEGORY-SPECIFIC EFFECTS}

The activation model revealed a significant main effect of grammatical category in seven clusters of contiguous voxels, including areas in the left posterior middle frontal gyrus, left posterior superior temporal sulcus/left middle temporal gyrus, left ventral temporal cortex, bilateral inferior parietal lobules, and bilateral occipital poles (Table 1; Figure 3).

We then interrogated these areas to determine whether they were preferentially activated for nouns or verbs, and whether these effects were modulated by lexical status, as described above. We found that three areas (left posterior middle frontal gyrus, left middle temporal gyrus, and left inferior parietal lobule) were activated more for verb trials than for noun trials. A fourth area (right inferior parietal lobule) also showed relatively greater activation for verb trials, but this difference did not survive correction for multiple comparisons in the post hoc analysis; we therefore did not consider this area to show significant category selectivity. One area (left ventral temporal cortex) showed greater activation for noun trials. In the remaining two areas (occipital poles), the apparent category effect was due to a significant decrease in activation for verb trials, while there was no change in the baseline for noun trials (Figure 4A).

In two areas (left inferior parietal lobule and left posterior middle frontal gyrus), the post hoc analysis also identified
Table 1 | Areas activated for nouns and verbs in the morphological transformation task ( $k=$ cluster size).

\begin{tabular}{|c|c|c|c|c|}
\hline & \multirow[t]{2}{*}{$\boldsymbol{k}$} & \multicolumn{3}{|c|}{ MNI coordinates } \\
\hline & & $x$ & $y$ & $z$ \\
\hline \multicolumn{5}{|l|}{ NOUN $\cup$ VERB } \\
\hline Left superior temporal gyrus & 10146 & -58 & -16 & 4 \\
\hline Left precentral gyrus & & -46 & -16 & 36 \\
\hline Right precentral gyrus & 10296 & 64 & -2 & 14 \\
\hline Right superior temporal gyrus & & 60 & -16 & -2 \\
\hline Left middle frontal gyrus & 445 & 36 & -52 & -18 \\
\hline Left occipital pole & 382 & -18 & -96 & -4 \\
\hline Left fusiform gyrus & & -30 & -78 & -10 \\
\hline Right fusiform gyrus & 661 & 36 & -52 & -18 \\
\hline Right occipital pole & & 18 & -94 & 2 \\
\hline Left anterior cingulate gyrus & 2067 & -6 & 19 & 30 \\
\hline Right anterior cingulate gyrus & & 6 & 22 & 38 \\
\hline Right posterior cingulate gyrus & 261 & 2 & -52 & 16 \\
\hline Right inferior parietal lobule & 186 & 40 & -58 & 54 \\
\hline Right middle occipital gyrus & 93 & 32 & -82 & 20 \\
\hline Right caudate & 224 & -12 & 0 & 2 \\
\hline Right putamen & 112 & 18 & 4 & 16 \\
\hline Right cerebellum & 5907 & 18 & -62 & -26 \\
\hline Left cerebellum & & -18 & -62 & 24 \\
\hline \multicolumn{5}{|l|}{ VERB $>$ NOUN } \\
\hline Left inferior parietal lobule & 204 & -44 & -40 & 50 \\
\hline Left middle/superior temporal gyrus & 272 & -54 & -40 & 4 \\
\hline Left middle frontal gyrus & 131 & -50 & 8 & 38 \\
\hline \multicolumn{5}{|l|}{ NOUN $>$ VERB } \\
\hline Left fusiform gyrus & 65 & -24 & -28 & -28 \\
\hline
\end{tabular}

Table 2 | Areas showing adaptation for nouns, verbs, or words of both categories in the morphological transformation task ( $k=$ cluster size).

\begin{tabular}{|c|c|c|c|c|}
\hline & \multirow[t]{2}{*}{$\boldsymbol{k}$} & \multicolumn{3}{|c|}{ MNI coordinates } \\
\hline & & $x$ & $y$ & $z$ \\
\hline \multicolumn{5}{|l|}{ NOUN UVERB } \\
\hline Left medial/superior frontal gyrus & 911 & -2 & 0 & 64 \\
\hline Left inferior frontal gyrus, pars opercularis & 385 & -52 & 8 & 10 \\
\hline Left middle frontal gyrus & 239 & -52 & 2 & 42 \\
\hline Left putamen & 97 & -22 & 0 & 6 \\
\hline Right putamen & 249 & 26 & 4 & -12 \\
\hline \multicolumn{5}{|l|}{ VERB $>$ NOUN } \\
\hline Left middle/inferior frontal gyrus & 60 & -52 & 24 & 24 \\
\hline
\end{tabular}

main effects of lexical status (real or pseudoword), with relatively greater activation for pseudoword trials than for real word trials. However, no significant interaction between lexical status and grammatical category was observed in any area. Overall, these findings are concordant with the results of an earlier study using the same paradigm (Shapiro et al., 2006), except for the occipital effect, which was not previously noted. 


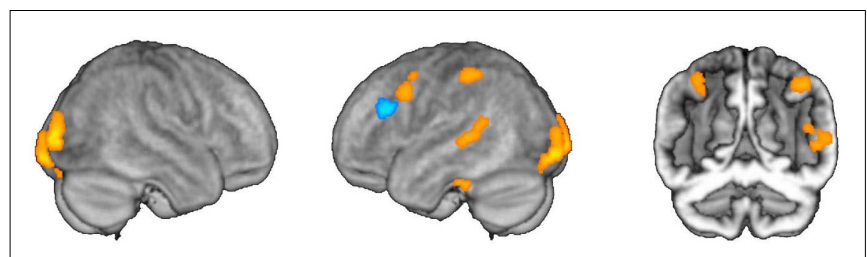

FIGURE 3 | Selective effects of activation and adaptation. Regions depicted in orange showed main effects of grammatical category in event-related activation. Regions depicted in blue showed main effects corresponding to adaptation for category-specific operations.

We then conducted an identical analysis for the adaptation model. Here, the results corresponding to the main effect of grammatical category were confined to an area near the junction of the left inferior and middle frontal gyri, which showed greater adaptation for verbs. There was no effect of lexical status, and no interaction between lexical status and grammatical category. No areas showed greater adaptation for nouns at the statistical thresholds we employed (Table 2; Figures 3 and 4B).

In a final analysis, we selected the four areas in which we observed grammatical category-selective activation in the activation model and adopted them as volumes of interest (VOIs) in the adaptation model, to determine whether these regions might show sub-threshold adaptation effects. (We did not include the areas in which categorical effects were driven exclusively by decreased activity.) No significant differences in "operation adaptation" across categories were observed within the regions that showed category specificity in the activation analysis (Figure 4B).

\section{DISCUSSION}

The combination of activation and operation-specific fMR adaptation analyses proved to be a potentially useful technique for identification areas selectively involved in the processing of nouns and verbs. Using a conventional activation model, we found that there was category-selective activation for verb production in the left posterior middle frontal gyrus, the left medio-lateral temporal cortex, and the left inferior parietal lobule. By contrast, noun production was associated with greater activation in the left fusiform gyrus. These results replicate the observations of an earlier fMRI study using a similar paradigm (Shapiro et al., 2006), and overlap in part with findings from other studies which employed a variety of production and comprehension tasks (Perani et al., 1999; Shapiro et al., 2005; Yokoyama et al., 2006; Palti et al., 2007; Bedny et al., 2008; Liljeström et al., 2008; Burton et al., 2009; Khader et al., 2010).

In addition, we found one brain area in which the amount of activation decreases over time for morphological transformations involving only one category of words, an effect we have called operation-specific adaptation. Interestingly, the operation-specific adaptation for verbs was observed in a more anterior portion of the middle frontal gyrus than the area in which activation for verbs was observed in this study and previous studies. However, previous work with rTMS has demonstrated that targeted suppression of this area results in selective interference for verbs in the same behavioral task used here (Shapiro et al., 2001; Cappelletti et al., 2008).
These findings have numerous limitations, not the least of which is that they rely on a specific and admittedly arbitrary assumption about the rate of adaptation. A more rigorous approach might involve analyzing the raw data on a subject-bysubject basis in order to derive empirically a function that describes changes in activation levels over time. On the other hand, assuming a simple linear adaptation parameter, as we have here, allows us to test hypotheses about the relationship between cognitive operations and neural activity using a technique that is easily implemented and not very computationally intensive.

With this caveat, we contend that the data presented here provide interesting insights into the neuroanatomical substrates of cognitive processes involved in language production. In general, the areas of category-general activation correspond to cortical regions engaged in various stages of word production, including the retrieval of phonological word forms, phonetic coding and articulation (Indefrey and Levelt, 2000), and lexical and semantic processing (Menenti et al., 2011). These processes are common to all tasks requiring spoken output.

By contrast, some of the areas in which strong operation adaptation was observed - namely, the left inferior frontal gyrus (Caplan, 2001; Friederici and Kotz, 2003; Menenti et al., 2011) and the striatum (Ullman et al., 1997; Friederici and Kotz, 2003; Longworth et al., 2005) - have been implicated specifically in the processing of phrase structure and grammatical inflection. Indeed, suppression of this region of the left inferior frontal gyrus with rTMS interferes with performance of this task for nouns and verbs equally (Cappelletti et al., 2008). The finding that these areas adapt to a task that emphasizes regular morphological affixation confirms the first prediction made in the Introduction, which was that operation-specific adaptation across categories should be found in parts of the brain that are generally involved in syntactically driven processes, including (but not necessarily limited to) morphological inflection. We did not observe adaptation for the morphological transformation task in brain areas thought to support other aspects of language production, such as the left temporal lobe.

Operation adaptation in the SMA was not predicted, but emerged quite robustly in the adaptation analysis. It is possible that this represents a general effect of cognitive adaptation, perhaps reflecting the reallocation of attentional resources as subjects became more practiced and familiar with the task (Rushworth et al., 2007). Aphasic patients with lesions in the SMA are reported to have difficulty initiating speech, but preserved fluency in repetition and in answering questions (Ziegler et al., 1997; Pai, 1999), an observation consistent with the idea that this region may indeed be less heavily recruited when a task is repeated or richly supported by the pragmatic context. On the other hand, there is some evidence that the medial SMA may be particularly important for the production of morphologically inflected forms (Sahin et al, 2006), and it may be that some portion of the area plays a critical role in selecting and encoding morphemes, including inflectional affixes (Alario et al., 2006).

The data also confirm our second prediction: the left midfrontal area found here to exhibit selective operation adaptation for verb trials corresponds to the area whose targeted suppression by TMS has been shown to result in a relative disruption in verb 


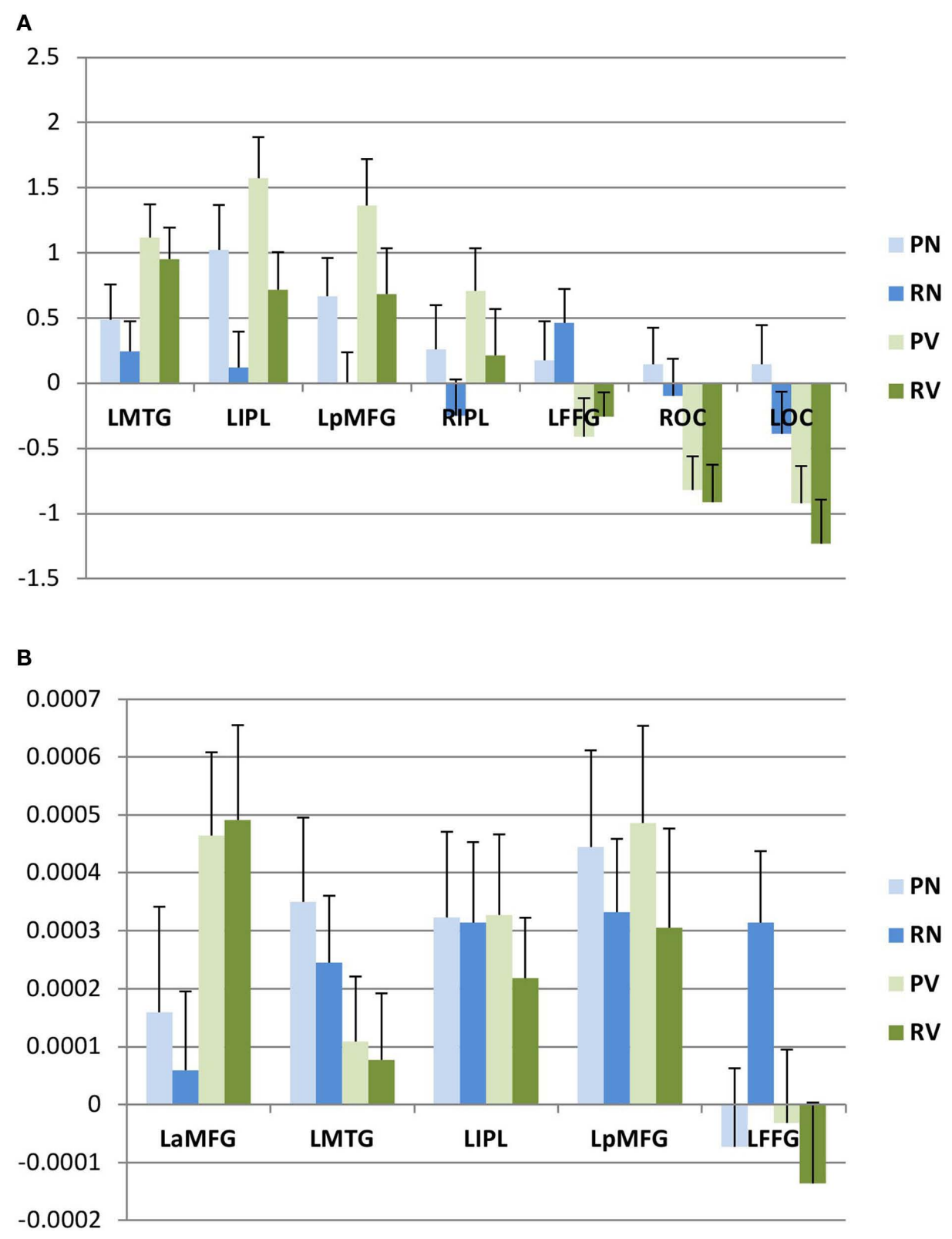

FIGURE 4 | Post hoc analysis. Within areas showing category-selective effects, we evaluated first eigenvariates for activation (A) and adaptation (B) by subject. Mean values (and standard errors of the mean) for these eigenvariates are plotted here. $L$, left; $R$, right; $B$, bilateral; a, anterior; $p$, posterior; MFG, middle frontal gyrus; IPL, inferior parietal lobule; MTG, middle temporal gyrus; FFG, fusiform gyrus; OC, occipital cortex. processing (Shapiro et al., 2001; Cappelletti et al., 2008). By contrast, TMS produces no category-specific effects when applied to the more posterior left frontal area that emerged in the conventional analysis (Cappelletti et al., 2008). This finding supports our hypothesis that brain areas crucial for the performance of a cognitive operation should exhibit hemodynamic adaptation as the task is repeated. The adaptation model appears to be both more sensitive and more specific than the (conventional) activation model for the identification of areas engaged in grammatical processing.

Here, we have defined the task of interest as selecting contextually appropriate inflectional forms of nouns and verbs. For verb trials, this depended on the computation of subject-verb agreement, which may be the function performed by the left anterior mid-frontal gyrus. We do not, however, claim that this area is necessarily specific for a particular morphosyntactic operation; another possibility is that this area is more generally engaged in the processing of verbs as defined by their syntactic context, which may include computation of agreement, tense, mood, and other properties that distinguish verbs from nouns in a given language (c.f. Shapiro et al., 2001). While these alternatives cannot be differentiated using the data at hand, it is clear that the region in question shows adaptation for a grammatical operation that 
applies to words independently of their meaning or phonological form.

Conversely, areas whose event-related response functions are similar across different cognitive operations may be engaged in processing features of stimuli that differ across trials independent of the specific operation performed in each trial. In the case at hand, this might include unique phonological, lexical, or semantic properties of words to be produced. For example, selective activation for nouns or verbs in areas putatively linked to semantic processing, such as the left medio-lateral temporal cortex, could reflect attempts to retrieve specific features of meaning within the broad domains of knowledge related to entities and events - arguably an automatic aspect of natural speech, though not the one this task was designed specifically to probe. Thus, more generally, different trends in event-related response

\section{REFERENCES}

Alario, F. X., Chainay, H., Lehericy, S., and Cohen, L. (2006). The role of the supplementary motor area (SMA) in word production. Brain Res. 1076, 129-143.

Bak, T. H., O’Donovan, D. G., Xuereb, J. H., Boniface, S., and Hodges, J. R. (2001). Selective impairment of verb processing associated with pathological changes in Brodmann areas 44 and 45 in the motor neurone diseasedementia-aphasia syndrome. Brain 124, 103-120.

Bedny, M., Caramazza, A., Grossman, E., Pascual-Leone, A., and Saxe, R. (2008). Concepts are more than percepts: the case of action verbs. J. Neurosci. 28, 11347-11353.

Berndt, R. S., Haendiges, A. N., and Burton, M. W. (2002). Grammatical class and imageability in aphasic word production: their effects are independent. J. Neurolinguistics 15, 353-371.

Burton, M. W., Krebs-Noble, D., Gullapalli, R. P., and Berndt, R. S. (2009). Functional neuroimaging of grammatical class: ambiguous and unambigious nouns and verbs. $\mathrm{Cogn}$. Neuropsychol. 26, 148-171.

Caplan, D. (2001). Functional neuroimaging studies of syntactic processing. J. Psycholinguist. Res. 30, 297-320.

Cappelletti, M., Fregni, F., Shapiro, K., Pascual-Leone, A., and Caramazza, A. (2008). Processing nouns and verbs in the left frontal cortex: a TMS study. J. Cogn. Neurosci. 20, 707-720.

Caramazza, A., and Hillis, A. E. (1991). Lexical organization of nouns and verbs in the brain. Nature 349, 788-790.

Crepaldi, D., Aggujaro, S., Arduino, L. S., Zonca, G., Ghirardi, G., Inzaghi, M. G., Colombo, M., Chierchia, G., and Luzzatti, C. (2006). Nounverb dissociation in aphasia: the role of imageability and functional locus of the lesion. Neuropsychologia 44 , 73-89.

Crepaldi, D., Berlingeri, M., Paulesu, E., and Luzzatti, C. (2011). A place for nouns and a place for verbs? A critical review of neurocognitive data on grammatical-class effects. Brain Lang. 116, 33-49.

Damasio, A. R., and Tranel, D. (1993). Nouns and verbs are retrieved with differently distributed neural systems. Proc. Natl. Acad. Sci. U.S.A. 90, 4957-4960.

Daniele, A., Giustolisi, L., Silveri, M. C., Colosimo, C., and Gainotti, G. (1994). Evidence for a possible neuroanatomical basis for lexical processing of nouns and verbs. Neuropsychologia 32, 1325-1341.

Dronkers, N. F., Pinker, S., and Damasio, A. R. (2000). "Language and the aphasias," in Principles of Neural Science, eds E. R. Kandel, J. H. Schwartz, and T. M. Jessell (New York, NY: McGraw-Hill), 1169-1187.

Faroqi-Shah, Y., and Thompson, C. K. (2004). Semantic, lexical, and phonological influences on the promatic aphasia. Brain Lang. 89, 484-498.

Francis, W. N., and Kucera, H. (1982). Frequency Analysis of English Usage. Boston: Houghton Mifflin Company.

Friederici, A. D., and Kotz, S. A. (2003). The brain basis of syntactic processes: functional imaging and lesion studies. Neuroimage 20, S8-S17.

Gold, B. T., Balota, D. A., Kirchhoff, B. A., and Buckner, R. L. (2005). Common and dissociable activation patterns associated with controlled semantic and phonological processing: evidence from fMRI adaptation. Cereb. Cortex 15, 1438-1450.

Goodglass, H. (1973). "Studies on the grammar of aphasics," in duction of verb inflections in agram-

functions may correspond to distinct levels of cognitive processing. If this is correct, the application of operation adaptation alongside content adaptation approaches and conventional eventrelated analyses of fMRI data may help to reveal hitherto obscure distinctions in the organization of neural networks that support human cognition.

\section{ACKNOWLEDGMENTS}

We thank T. Daley for assistance in collecting data; S. Slotnick for critical discussions; and R. Buckner, D. Caplan, D. Holtzman, and J. Schwarzbach for review of the manuscript. The authors are funded by the National Institute on Deafness and Other Communication Disorders (Alfonso Caramazza, Lauren R. Moo) and by the National Institute of Neurological Disorders and Stroke (Kevin A. Shapiro).

Psycholinguistics and Aphasia, eds $\mathrm{H}$. Goodglass and S. Blumstein (Baltimore, MD: Johns Hopkins University Press), 183-215.

Grill-Spector, K., Henson, R., and Martin, A. (2006). Repetition and the brain: neural models of stimulusspecific effects. Trends Cogn. Sci. (Regul. Ed.) 10, 14-23.

Henson, R., Shallice, T., and Dolan, R. (2000). Neuroimaging evidence for dissociable forms of repetition priming. Science 287, 1269-1272.

Indefrey, P., and Levelt, W. J. M. (2000). "The neural correlates of language production," in The New Cognitive Neurosciences, ed. M. Gazzaniga (Cambridge, MA: MIT Press), 845-865.

Kean, M.-L. (1978). The linguistic interpretation of aphasic syndromes: agrammatism in Broca's aphasia, an example. Cognition 5, 9-46.

Khader, P. H., Jost, K., Mertens, M., Bien, S., and Rösler, F. (2010). Neural correlates of generating visual nouns and motor verbs in a minimal phrase context. Brain Res. 1318 122-132.

Laiacona, M., and Caramazza, A. (2004). The noun/verb dissociation in language production: varieties of causes. Cogn. Neuropsychol. 21, 103-124.

Liljeström, M., Tarkiainen, A., Parviainen, T., Kujala, J., Numminen, J., Hiltunen, J., Laine, M., and Salmelin, R. (2008). Perceiving and naming actions and objects. Neuroimage 41 , 1132-1141.

Longe, O., Randall, B., Stamatakis, E. A., and Tyler, L. K. (2007). Grammatical categories in the brain: the role of morphological structure. Cereb. Cortex 17, 1812-1820.

Longworth, C. E., Keenan, S. E., Barker, R. A., Marslen-Wilson, W. D., and Tyler, L. K. (2005). The basal ganglia and rule-governed language use: evidence from vascular and degenerative conditions. Brain 2005, 584-596.

Luzzatti, C., Aggujaro, S., and Crepaldi, D. (2006). Verb-noun double dissociation in aphasia: theoretical and neuroanatomical foundations. Cortex $42,875-883$.

Marslen-Wilson, W., and Tyler, L. K. (1997). Dissociating types of mental computation. Nature 387 , 592-594.

Martin, A., Wiggs, C. L., and Weisberg, J. (1997). Modulation of human medial temporal lobe activity by form, meaning, and experience. Hippocampus 7, 587-593.

McCarthy, R. A., and Warrington, E. K. (1985). Category specificity in an agrammatic patient: the relative impairment of verb retrieval and comprehension. Neuropsychologia 23, 709-727.

Menenti, L., Gierhan, S. M. E., Segaert, K., and Hagoort, P. (2011). Shared language: overlap and segregation of the neuronal infrastructure for speaking and listening revealed by functional MRI. Psychol. Sci. 22, 1173-1182.

Miceli, G., Silveri, M. C., Romani, C., and Caramazza, A. (1989). Variation in the pattern of omissions and substitutions of grammatical morphemes in the speech of so-called agrammatic patients. Brain Lang. 36, 447-492.

Miceli, G., Silveri, M. C., Villa, G., and Caramazza, A. (1984). On the basis for the agrammatic's difficulty in producing main verbs. Cortex 20, 207-220.

Obler, L. K., Harris, K., Meth, M., Centeno, J., and Mathews, P. (1999). The phonology-morphosyntax interface: affixed words in agrammatism. Brain Lang. 68, 233-240.

Pai, M. C. (1999). Supplementary motor area aphasia: a case report. Clin. Neurol. Neurosurg. 101, 29-32. 
Palti, D., Ben Shachar, M., Hendler, T., and Hadar, U. (2007). Neural correlates of semantic and morphological processing of Hebrew nouns and verbs. Hum. Brain Mapp. 28, 303-314.

Perani, D., Cappa, S. F., Schnur, T., Tettamanti, M., Collina, S., Rosa, M. M., and Fazio, F. (1999). The neural correlates of noun and verb processing: a PET study. Brain 122, 2337-2344.

Rushworth, M. F., Buckley, M., Behrens, T., Walton, M., and Bannerman, D. (2007). Functional organization of the medial frontal cortex. Curr. Opin. Neurobiol. 17, 220-227.

Sahin, N. T., Pinker, S., and Halgren, E. (2006). Abstract grammatical processing of nouns and verbs in Broca's area: evidence from fMRI. Cortex 42 , 540-562.

Shapiro, K., and Caramazza, A. (2003). Grammatical processing of nouns and verbs in left frontal cortex? Neuropsychologia 41, 1189-1198.

Shapiro, K., Shelton, J., and Caramazza, A. (2000). Grammatical class in lexical production and morphological processing: evidence from a case of fluent aphasia. Cogn. Neuropsychol. 17, 665-682.

Shapiro, K. A., Moo, L. R., and Caramazza, A. (2006). Cortical signatures of noun and verb production.
Proc. Natl. Acad. Sci. U.S.A. 103, 1644-1649.

Shapiro, K. A., Mottaghy, F. M., Schiller, N. O., Poeppel, T. D., Flüss, M. O., Müller, H.-W., Caramazza, A., and Krause, B. J. (2005). Dissociating neural correlates for nouns and verbs. Neuroimage 24, 1058-1067.

Shapiro, K. A., Pascual-Leone, A., Mottaghy, F. M., Gangitano, M., and Caramazza, A. (2001). Grammatical distinctions in the left frontal cortex. J. Cogn. Neurosci. 13, 713-720.

Slotnick, S. D., Moo, L. R., Segal, J. B., and Hart, J. Jr. (2003). Distinct prefrontal cortex activity associated with item memory and source memory for visual shapes. Brain Res. Cogn. Brain Res. 17, 75-82.

Toni, I., Krams, M., Turner, R., and Passingham, R. E. (1998). The time course of motor sequence learning: a whole-brain fMRI study. Neuroimage 8, 50-61.

Tyler, L. K., Bright, P., Fletcher, P., and Stamatakis, E. A. (2004). Neural processing of nouns and verbs: the role of inflectional morphology. Neuropsychologia 42, 512-523.

Tyler, L. K., Randall, B., and Stamatakis, E. A. (2008). Cortical differentiation for nouns and verbs depends on grammatical markers. J. Cogn. Neurosci. 20, 1381-1389.
Tyler, L. K., Stamatakis, E. A., Post, B., Randall, B., and Marslen-Wilson, W. (2005). Temporal and frontal systems in speech comprehension: an fMRI study of past tense processing. Neuropsychologia 43, 1963-1974.

Ullman, M., Corkin, S., Coppola, M., Hickok, G., Growdon, J., Koroshetz, W., and Pinker, S. (1997). A neural dissociation within language: evidence that the mental dictionary is part of declarative memory, and that grammatical rules are part of the procedural system. J. Cogn. Neurosci. 9, 266-276.

van Turrennout, M., Bielamowicz, L., and Martin, A. (2003). Modulation of neural activity during object naming: effects of time and practice. Cereb. Cortex 13, 381-391.

Wagner, A. D., Koutstall, W., Maril, A., Schacter, D. L., and Buckner, R. L. (2000). Task-specific repetition priming in left inferior prefrontal cortex. Cereb. Cortex 10 1176-1184.

Yokoyama, S., Miyamoto, T., Riera, J., Kim, J., Akitsuki, Y., Iwata, K., Yoshimoto, K., Horie, K., Sato, S., and Kawashima, R. (2006). Cortical mechanisms involved in the processing of verbs: an fMRI study. J. Cogn Neurosci. 18, 1304-1313.
Ziegler, W., Kilian, B., and Deger, K. (1997). The role of the left mesial frontal cortex in fluent speech: evidence from a case of left supplementary motor area hemorrhage. Neuropsychologia 35, 1197-1208.

Conflict of Interest Statement: The authors declare that the research was conducted in the absence of any commercial or financial relationships that could be construed as a potential conflict of interest.

Received: 29 March 2011; accepted: 19 January 2012; published online: 08 February 2012.

Citation: Shapiro KA, Moo LR and Caramazza A (2012) Neural specificity for grammatical operations is revealed by content-independent $f M R$ adaptation. Front. Psychology 3:26. doi: 10.3389/fpsyg.2012.00026

This article was submitted to Frontiers in Language Sciences, a specialty of Frontiers in Psychology.

Copyright (C) 2012 Shapiro, Moo and Caramazza. This is an open-access article distributed under the terms of the Creative Commons Attribution Non Commercial License, which permits noncommercial use, distribution, and reproduction in other forums, provided the original authors and source are credited. 